\title{
Spectroscopy of an intrinsic fluorophore in animal and plant milk for potential identification of their quality
}

\author{
Lopamudra Roy, ${ }^{1,2}$ Animesh Halder, ${ }^{1,2}$ Soumendra Singh, ${ }^{3,4}$ Jayita Patwari, ${ }^{3}$ Priya Singh, ${ }^{3}$ \\ Kallol Bhattacharya, ${ }^{2}$ Soumen Mondal, ${ }^{1,5 *}$ and Samir Kumar Pal ${ }^{1,3 *}$ (D) \\ ${ }^{1}$ Technical Research Centre, S. N. Bose National Centre for Basic Sciences, Block JD, Sector III, Salt Lake, Kolkata: 700 106, India \\ ${ }^{2}$ Department of Applied Optics and Photonics, University of Calcutta, JD-2, Sector-III, Salt Lake, Kolkata: 700 106, India \\ ${ }^{3}$ Department of Chemical, Biological and Macromolecular Sciences, S. N. Bose National Centre for Basic Sciences, Block JD, Sector III, \\ Salt Lake, Kolkata: 700 106, India \\ ${ }^{4}$ Center for Astroparticle Physics and Space Science, Bose Institute, Sector III, Salt Lake, Kolkata: 700091, India \\ ${ }^{5}$ Department of Astrophysics and Cosmology, S. N. Bose National Centre for Basic Sciences, JD Block, Sector III, Salt Lake, Kolkata: \\ 700 106, India
}

\section{ABSTRACT}

Riboflavin (RF), commonly known as vitamin $\mathrm{B}_{2}$, is an essential ingredient in any milk variety of animal origin. The photophysics of the molecule RF, including its interaction with biological macromolecules, are well studied. Here, we have investigated the possibility of the molecule as a potential biomarker of milk quality. We also found omnipresence of this molecule in milk of plant origin (soy milk). Spectroscopic studies on various animal and plant milks of different commercial origins confirmed the potential of RF for use in identifying the quality of the milk varieties. Our developed strategy involved identification or spectroscopic signature of RF by measuring optical density at $365 \mathrm{~nm}$ (quality factor 1) and fluorescence intensity around $520 \mathrm{~nm}$ (excitation at $365 \mathrm{~nm}$; quality factor 2) on a very small amount of whole milk $(10 \mu \mathrm{L})$. We also developed a prototype device called Mil-Q-Way to be used in the real field. The required interfacing software in the LabView platform was also developed. A 2-parameter plot (quality factor 1 on the $\mathrm{x}$-axis and quality factor 2 on the y-axis) called the Mil-Q-Way plot clearly differentiates the quality of milks of different commercial origins. The low-cost device based on simple spectroscopy was shown to screen for the presence of harmful adulterants in drinkable milk.

Key words: milk, biomarker, riboflavin, vitamin $\mathrm{B}_{2}$, fluorescence spectroscopy

\footnotetext{
Received May 29, 2019.

Accepted October 7, 2019.

*Corresponding authors: skpal@bose.res.in and soumen.mondal@ bose.res.in
}

\section{INTRODUCTION}

A classic example of a balanced food is milk. Milk primarily consists of water, protein, fat, lactose, vitamins, and ash (Reddy et al., 2000; Cheung and Mehta, 2015; Poonia et al., 2017). Among the macro- and micronutrient molecules in milk, vitamin $\mathrm{B}_{2}$, or riboflavin $(\mathbf{R F})$, is an important one (Powers, 2003). The quantity of RF ranges from 0.17 to $0.20 \mathrm{mg} / 100 \mathrm{~g}$ in cow milk and varies from breed to breed. It helps to maintain normal biofunctioning of energy-yielding metabolism, the nervous system, and so on (Muehlhoff et al., 2013). The nutritional value and cost of milk compared with that of other healthy foods has made it a significant component of the diets of many populations worldwide. However, the increased demand on the milk supply globally can lead to massive levels of fraudulent activity (Salih and Yang, 2017). The nutritional value of milk gets lessened due to the illegal addition of adulterants for financial profit (Navale and Gupta, 2016) or a lack of concern about nonhygenic conditions that can make the milk a high-risk commodity. Water is the most commonly used adulterant for milk, which reduces the food's nutritional value (Barham et al., 2014; Pradeep et al., 2016). Sometimes the added water itself is unhygienic because it may contain toxic adulterants (Navale and Gupta, 2016). Additionally, the decreased SNF content and nutritional value due to addition of water enhances the risk of health hazards and degrades the quality of the milk (Handford et al., 2016).

Several standard technologies are being developed to detect adulteration in milk. A sensing-based development using single-frequency electrical conductance measurement uses the cryoscopic method to detect added water in full-fat milk by measuring the changes in conductance (Mabrook and Petty, 2003). Enzymelinked immunosorbent assay is a widely used technique for determining the authenticity (Asensio et al., 2008) of 
a large number of samples and is cheaper and less time consuming compared with PCR, which is a DNA-based method also used in determination of food authenticity. Enzyme-linked immunosorbent assay (Hurley et al., 2004, 2006) and HPLC with electrospray ionization MS (Chen et al., 2004) are capable of detecting adulteration in very similar kinds of milks, such as cow and buffalo or goat and sheep. Milk fat adulteration can be analyzed using gas chromatography (Lipp, 1995). Milk quality has been previously classified using low-cost optical capillaries (Borecki et al., 2009). Another method for detecting raw milk adulteration is MS, in which the MALDI-TOF method is used. $\alpha$-Lactalbumin whey protein is considered a biomarker of raw milk in this method (Cozzolino et al., 2001). Near-infrared (Huang et al., 2008) and mid-infrared (De Marchi et al., 2009) spectroscopy techniques are widely used in food and dairy product industries. Previous studies have used the infrared spectroscopic technique to determine melamine in milk (Jawaid et al., 2013) and quality of milk (Bittante et al., 2012).

Compared with the above-mentioned techniques, fluorescence spectroscopy is less expensive and relatively easier because it does not require rigorous sample preparation. In some earlier reports, cow milk adulteration in buffalo milk was identified using the fluorescence intensity of the biomarkers $\beta$-carotene and vitamin $\mathrm{A}$ (Ullah et al., 2017). Adulteration of milk fat was detected in another report on the basis of changes in the optical characteristics of 3 fluorophore markers (tryptophan, tocopherol, and RF; Ntakatsane et al., 2013). In other reports in which the fluorescence intensity of RF was used to detect the quality, packaging condition, and freshness of yogurt, a decrease in fluorescence intensity of the biomarker RF was correlated with the degradation of the quality of the yogurt (Miquel Becker et al., 2003). The use of RF as a fluorescence marker has been reported earlier, but the method is quite different from ours. Changes in both fluorescence intensity of RF and optical density of milk give us information about adulteration in milk.

In the present work, $\mathrm{RF}$, the intrinsic fluorophore of milk, is shown to be a potential biomarker of quality identification of milk from different farms. Detailed optical spectroscopic in vitro and in vivo (in milk) studies of RF in various environments have been carried out to give enough proof and to establish it as the potential biomarker. We have found that overall optical density at $365 \mathrm{~nm}$ represents the colloidal density of milk, which is considered to be quality factor 1 (Q1). On the other hand, the fluorescence intensity of milk at $520 \mathrm{~nm}$ (excitation at $365 \mathrm{~nm}$ ) is considered to be quality factor 2 (Q2), which indicates the concentration of the biomarker in the milk variety. The interface of the prototype device has been made in the LabVIEW platform (http://www.ni.com/en-in/about-ni.html) for the identification of the origin of milks, including plant varieties from various companies. The sensing device is named Mil-Q-Way throughout this article for ease of reading. "Mil" stands for "measurement," "Q" stands for "quality," and "Way" stands for "time during the retailing process." The measurement of the milk quality that might get adulterated during the retail process was the idea behind such a nomenclature. The introduction of Q1 and Q2 for analysis of milk quality provides the novelty of the present invention and helps to distinguish our invention from the other spectroscopy-based techniques of milk quality detection. Our method, which detects 2 intrinsic properties of the milk components, is more reliable than the methods of detection that deal with only 1 parameter. To our understanding, the developed spectroscopic strategy would be useful for the sensitive detection of milk adulteration.

\section{MATERIALS AND METHODS}

\section{Reagents}

Riboflavin, BSA, and L- $\alpha$-phosphatidylcholine (PC) were obtained from Sigma-Aldrich (St. Louis, MO). Different types of milks of animal and plant origin were obtained from different commercial firms. Our milk identification library contains milks of various sources, such as Go Daily Milk (Parag Milk Foods Ltd., Andhra Pradesh, India), Soy Milk (Hershey India Privet Limited, MP, India), Amul Standardize Milk (Amul, Gujrat, India), Amul Toned Milk (Amul), and Express Dairy Milk (Express Dairy Company Ltd., Kolkata, India).

\section{Preparation of Samples}

A $1 \mu M \mathrm{RF}$ solution was prepared in dimethyl sulfoxide, and $100 \mu M$ solutions of BSA, PC, and BSA-PC mixture were prepared in $50 \mathrm{~m} M$ phosphate buffer using water from a Millipore system (Millipore, Burlington, MA). Different milk samples were prepared by adding $10 \mu \mathrm{L}$ of milk to $2 \mathrm{~mL}$ of distilled water. For the preparation of adulterated milk stocks, $68 \mathrm{mg}$ of urea, $200 \mathrm{mg}$ of sucrose, and $50 \mu \mathrm{L}$ of vegetable oil in $1 \mathrm{~mL}$ of milk were used. Samples for the experiment were prepared by taking $10 \mu \mathrm{L}$ of adulterated milk from the stock in $2 \mathrm{~mL}$ of distilled water.

\section{Development of Low-Cost Instrument and Working Principle}

Figure 1a shows a diagram of the Mil-Q-Way, and Figure $1 \mathrm{~b}$ shows a cross-sectional view of its setup. 


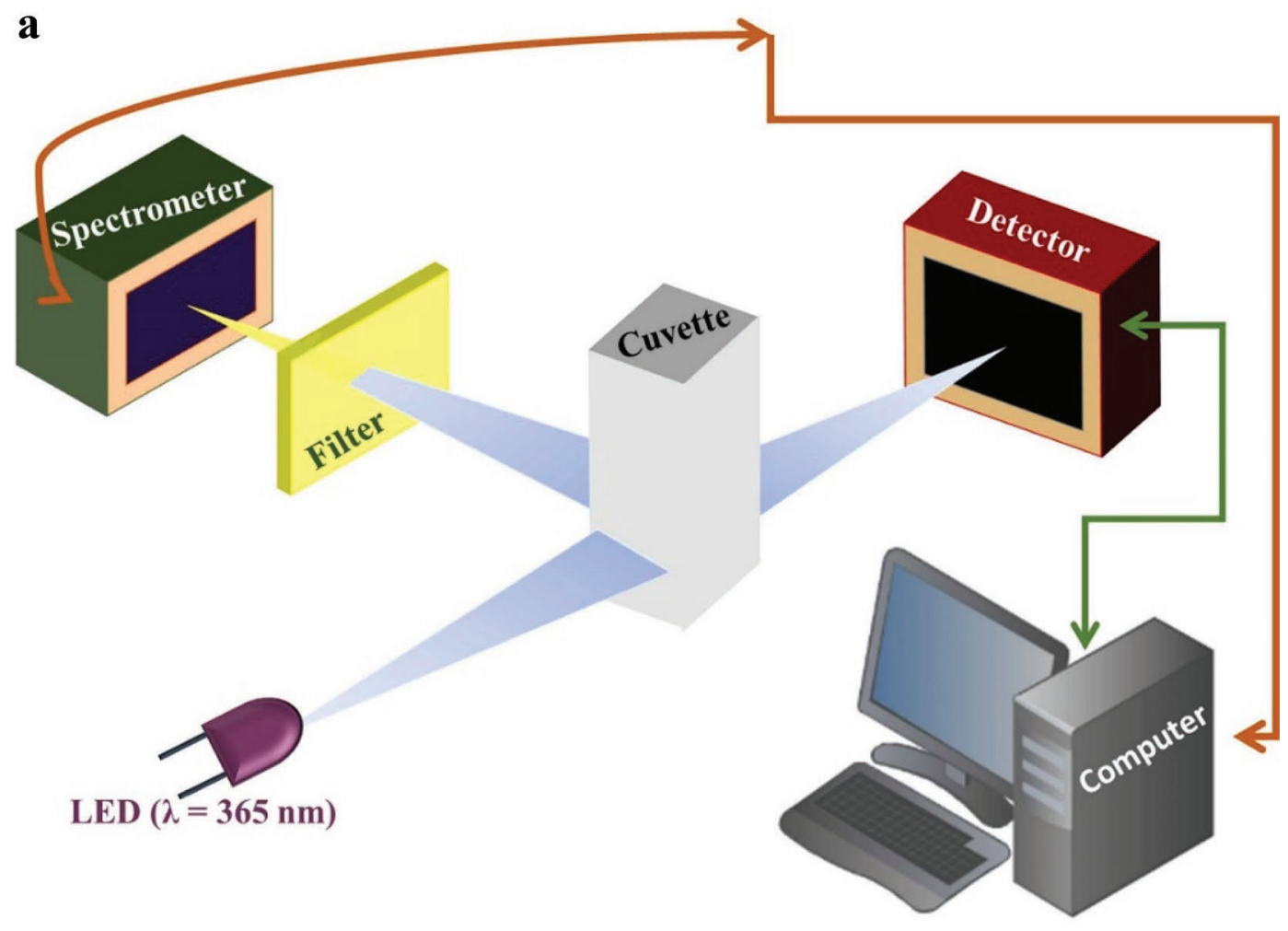

b

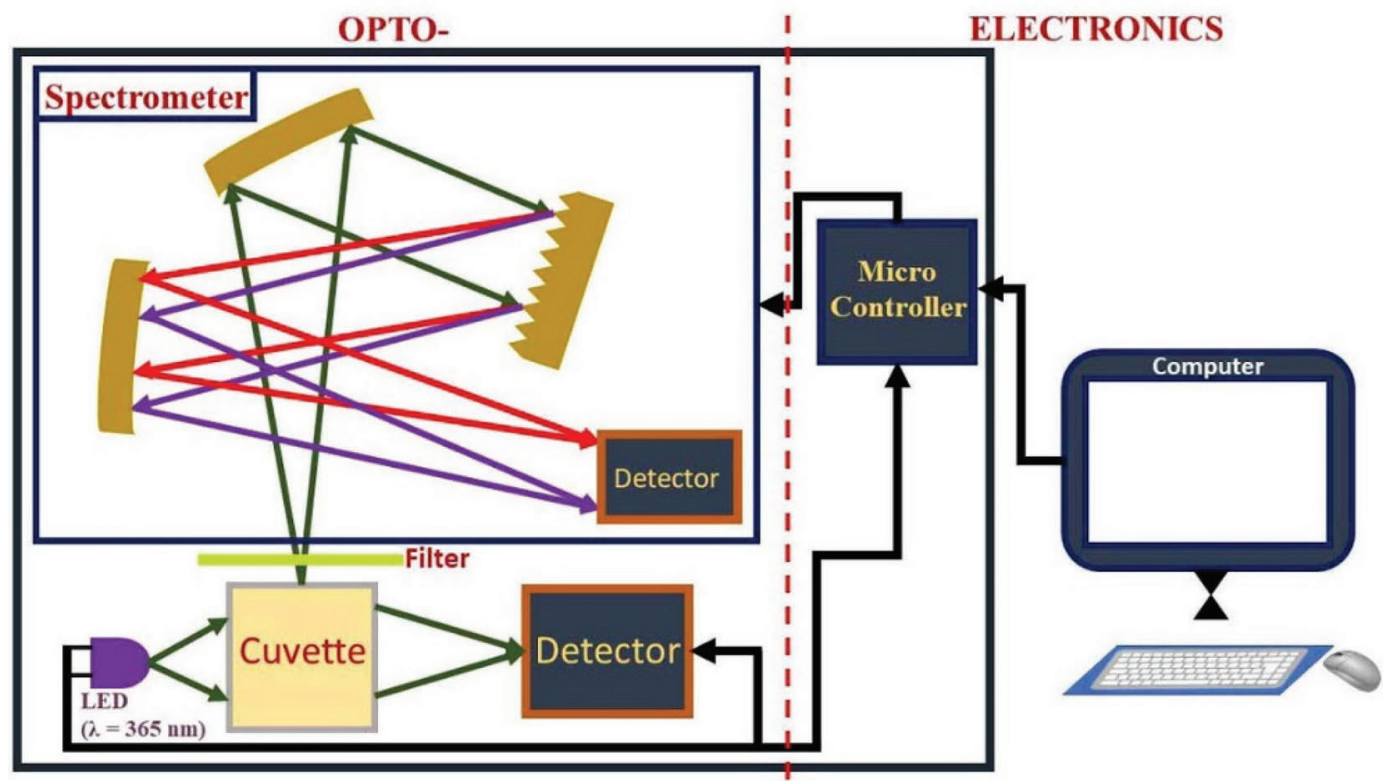

Figure 1. (a) Diagram of the Mil-Q-Way and (b) cross-sectional view of its setup. Source: UV light-emitting diode (LED) at 365 nm (2 A maximum, 15 V DC). Detector: photodiode (Adafruit TSL2561, Adafruit Industries, New York, NY) with current ranging from $15 \mu$ A (powerdown mode) to $0.5 \mathrm{~mA}$ (active-sensing mode). Filter: high-pass filter $\left(\lambda_{\text {pass }}>400 \mathrm{~nm}\right.$, where $\lambda_{\text {pass }}$ is the wavelength at which the transmission increases to 50\% throughput in the high-pass filter). Spectrometer: Black Comet (StellarNet Inc., Keystone, FL; wavelength range $=200-1,080$ $\mathrm{nm}$; dynamic range $=2,000: 1$ with 6 decades and a charge-coupled device-based detector with 2,048 pixels). 


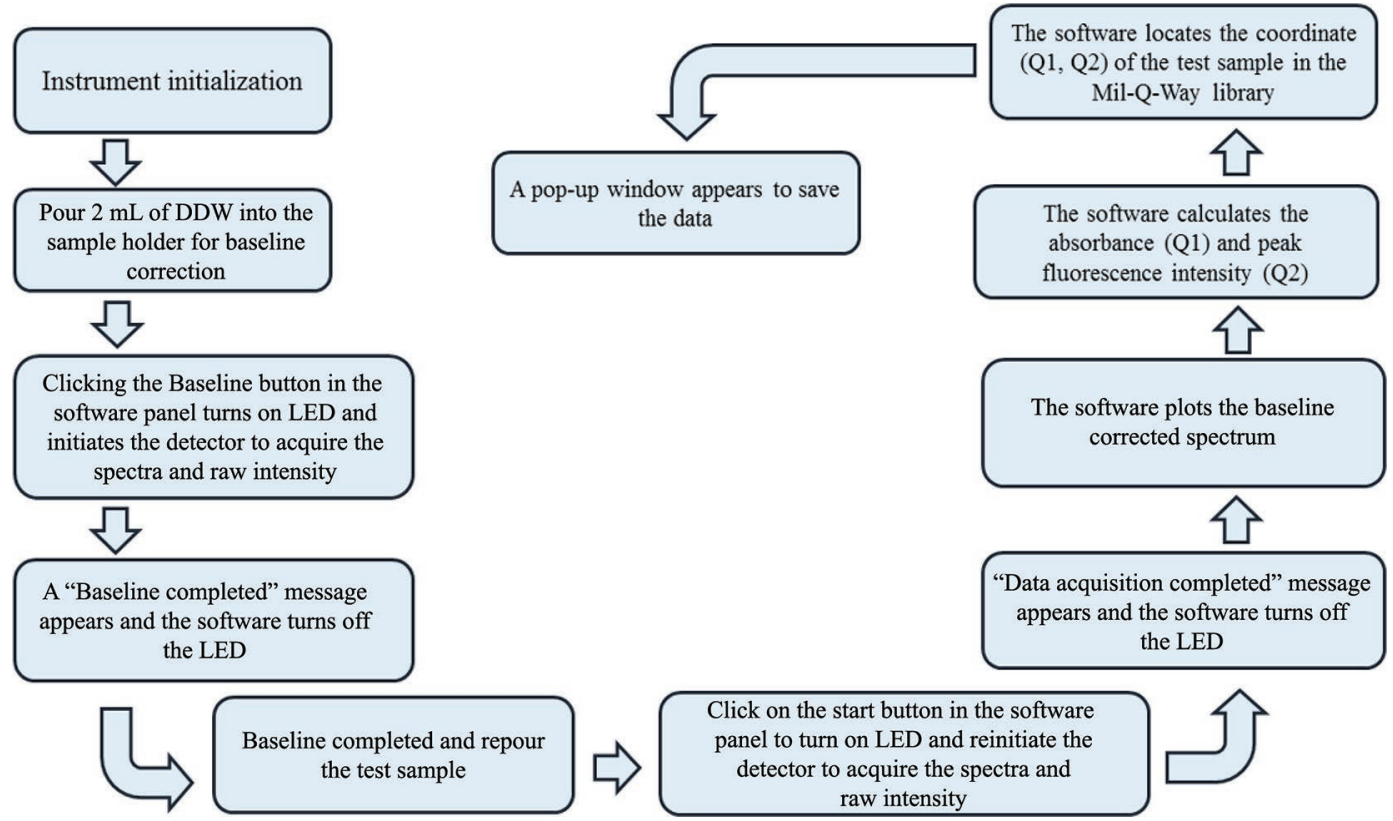

Figure 2. Flow chart of the experiment. DDW = double-distilled water; LED = light-emitting diode.

The working principle of the instrument is based on the well-known Beer-Lambert law. A 365-nm UV lightemitting diode (LED; 2 A maximum, $15 \mathrm{~V}$ DC) is used as the light source to excite the sample. The fluorescence is collected at a $90^{\circ}$ angle compared with the excited light beam using a spectrometer (Black-Comet, StellarNet Inc., Keystone, FL; wavelength range = 200-1,080 nm; dynamic range $=2,000: 1$ with 6 decades and a charge-coupled device-based detector with 2,048 pixels), and absorption of the sample is collected using a photodetector [Adafruit TSL2561, Adafruit Industries, New York, NY, with current ranging from $15 \mu \mathrm{A}$ (power-down mode) to $0.5 \mathrm{~mA}$ (active-sensing mode)] in the direction of the excited light beam. A high-pass filter $\left(\lambda_{\text {pass }}>400 \mathrm{~nm}\right.$, where $\lambda_{\text {pass }}$ is the wavelength at which the transmission increases to $50 \%$ throughput in the high-pass filter) is used to absorb the excitation wavelength to reduce the scattering in the fluorescence collection path. The spectrometer, detector, data collection, and processing are controlled using a microcontroller, whereby the LabView interfacing program connects the instrument with the computer system.

\section{Interfacing Software Design}

A LabView-based program is designed to acquire and process data from the instrument via a USB port. A Windows (Microsoft Inc., Redmond, WA)-based onboard computer is used to run the developed software in real time to acquire data. The interface is made to be simple and intuitive. Thus, running the software does not require additional training. After the program is started, it automatically checks the availability and condition of the inbuilt sensors and light sources. If any problem is detected, it terminates itself by prompting an error message. If everything is found to be in an acceptable condition, the program asks the user to provide a sample and waits for the desired action. After the user clicks OK, the UV LED is switched on, and collection of the optical density and fluorescence data is initiated by activating the spectrometer and the photodiode. The same action is repeated for other samples. The data are collected and stored in simple text files for offline analysis, if required.

\section{Data Processing}

To determine the quality of the unknown sample, the instrument must have prior information about a sample of known quality. All of the results obtained for unknown samples in our device are compared with the preloaded results of the known samples. A preloaded data gallery, or soft library, for milk quality detection is an integral part of the instrument. To create the soft library, a sample of one particular commercial origin is tested 5 times, and the Q1 and Q2 values are stored in memory. Fluctuation of the Q1 and Q2 is noted to define a specific area on the Q1-Q2 plot for locating that particular milk. Using the same principle, milks of 6 different commercial origins are localized in the 

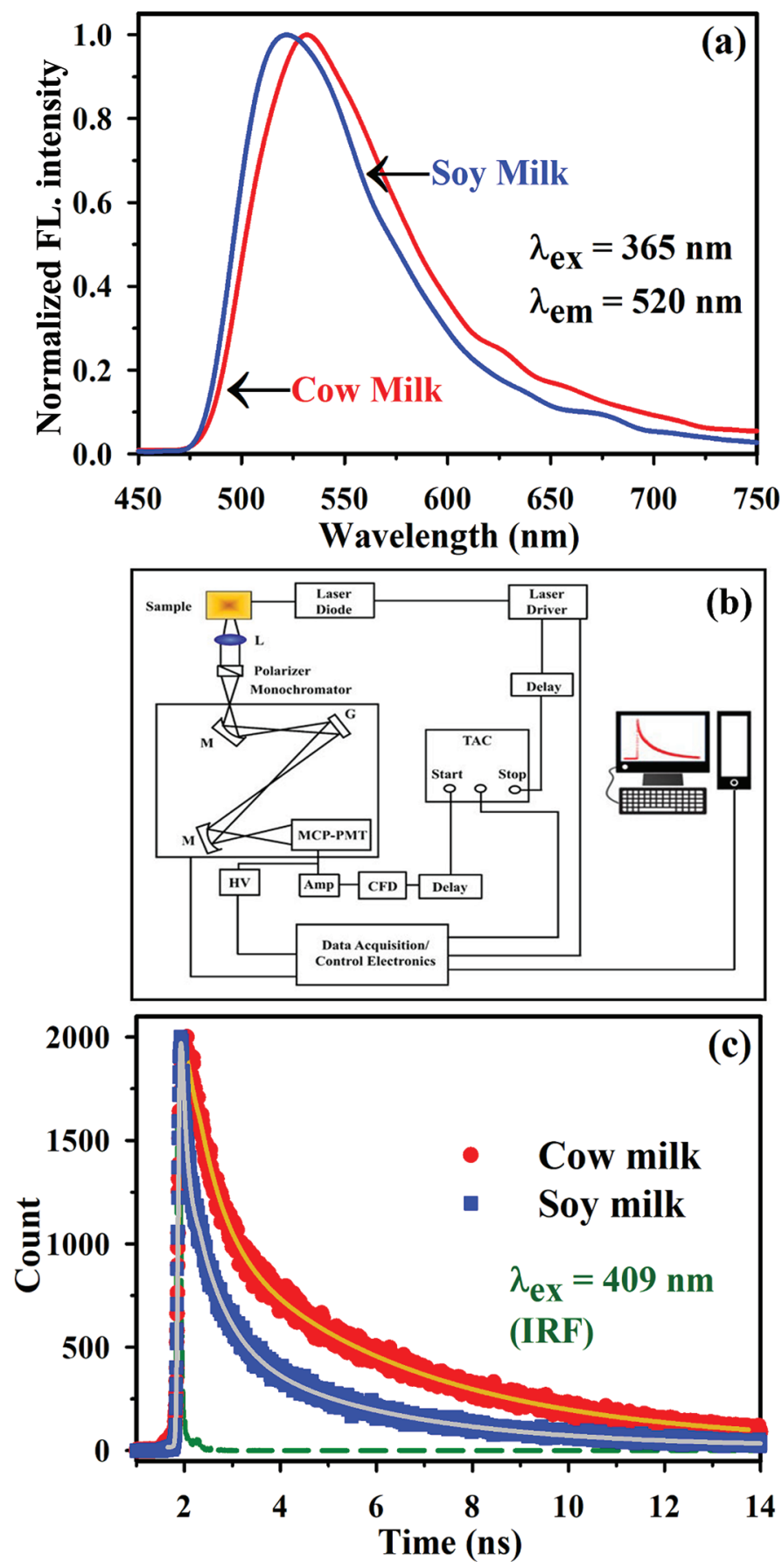

Figure 3. (a) Emission spectra of cow and soy milks showing fluorescence (FL.) peak at around $520 \mathrm{~nm}$. (b) Diagram of the timecorrelated single-photon counting (TCSPC) spectrometer. L = lens; $\mathrm{M}=$ mirror; $\mathrm{G}=$ grating; HV high-voltage source; MCP-PMT = microchannel plate photomultiplier tube; $\mathrm{TAC}=$ time to amplitude converter; $\mathrm{CFD}=$ constant fraction discriminator; Amp = amplification. (c) Picosecond time-resolved decays of cow and soy milks using the TCSPC spectrometer. Count $=$ emission count; IRF $=$ instrument response function; $\lambda_{\mathrm{em}}=$ emission wavelength; $\lambda_{\mathrm{ex}}=$ excitation wavelength.
Q1-Q2 plot; this is considered the reference library in our proposed method. The soft library could be modified according to the aim of the work.

The software calculates the parameters, Q1 and Q2, of unknown milk. After calculation, the software compares these values with a set of preinstalled values from the individual categories of milks available in the library. The data are then plotted online to get the quality index, which indicates their position compared with other available data for samples of known quality. This indexing helps a nonqualified person to estimate the quality of the sample being tested. The software is designed in such a way that after successful completion of the test for a single sample, it automatically asks for the next sample. Figure 2 summarizes the flow of working and data processing.

\section{Optical Characterization}

Absorption spectra of samples were recorded using a UV-2600 Shimadzu spectrometer (Shimadzu Corp., Kyoto, Japan) in the wavelength range of 400 to 700 $\mathrm{nm}$. The steady-state emission and excitation spectra were recorded using a Fluorolog fluorimeter (Horiba Jobin Yvon, Irvine, CA). A time-correlated singlephoton counting setup from Edinburgh Instruments (Livingston, UK) was used for time-resolved spectroscopic measurements (see Figure 3b for diagram). A picosecond pulsed laser of $409 \mathrm{~nm}$ was the light source, and instrument response function was 90 ps during measurement. The working principle of the instrument has been discussed in detail previously (Lakowicz, 2013). According to the diagram, the start channel of the time to amplitude converter connects the signal coming from the microchannel plate photomultiplier tube via constant fraction discriminator after amplification. Another delay line connects the laser with the stop channel of the time to amplitude converter. All the samples were taken in solvent water during measurement.

\section{RESULTS AND DISCUSSION}

Figure 3a shows the normalized steady-state emission spectra of the animal and plant milks. The fluorescence peak appearing at around $520 \mathrm{~nm}$ indicates the possibility of the presence of RF molecules in the milk samples of different origins. Comparing the emission spectrum of the milk samples with that of the literature values of $R F$, it is evident that $R F$ is one of the intrinsically present fluorophores of milk. Figure $3 \mathrm{~b}$ shows a diagram of the time-correlated single-photon counting spectrometer. This instrument was used to collect the time-resolved fluorescence decay information of the 
Table 1. Excited-state lifetime of the different samples

\begin{tabular}{|c|c|c|c|c|c|}
\hline Sample $^{1}$ & $\begin{array}{c}\text { Excitation } \\
\text { wavelength }(\mathrm{nm})\end{array}$ & $\begin{array}{c}\text { Emission } \\
\text { wavelength }(\mathrm{nm})\end{array}$ & $\tau_{1}[\mathrm{~ns}(\%)]$ & $\tau_{2}[\mathrm{~ns}(\%)]$ & $\tau_{3}[\mathrm{~ns}(\%)]$ \\
\hline Soy milk & 409 & 520 & $0.08(55)$ & $0.63(29)$ & $3.55(15.9)$ \\
\hline Cow milk & 409 & 520 & $0.52(54.26)$ & $4.24(45.74)$ & \\
\hline $\mathrm{RF}+\mathrm{BSA}$ & 409 & 520 & $0.07(46.14)$ & $0.71(18.89)$ & $4.25(34.97)$ \\
\hline $\mathrm{RF}+\mathrm{PC}$ & 409 & 520 & $0.43(20.29)$ & $4.25(79.71)$ & \\
\hline $\mathrm{RF}+\mathrm{BSA}+\mathrm{PC}$ & 409 & 520 & $0.06(48.34)$ & $0.67(20.46)$ & $4.25(31.2)$ \\
\hline
\end{tabular}

${ }^{1} \mathrm{RF}=$ riboflavin; $\mathrm{PC}=\mathrm{L}-\alpha-$ phosphatidylcholine; $\tau_{1}, \tau_{1}$, and $\tau_{3}=3$ different time scales corresponding to the fitting of the fluorescence decays

samples in the present work. Figure 3c shows timeresolved photoluminescence of milk samples of different origins. The corresponding excited state lifetime of the fluorophores present in milk samples is tabulated in Table 1, which clearly indicates that the longer time component (time scale corresponding to the fitting of the fluorescence decay) of milk samples is similar to the reported values of radiative lifetime for $\mathrm{RF}$ using a 409-nm light source (Chaudhuri et al., 2015). In building the Mil-Q-Way, a 365-nm UV light source was used
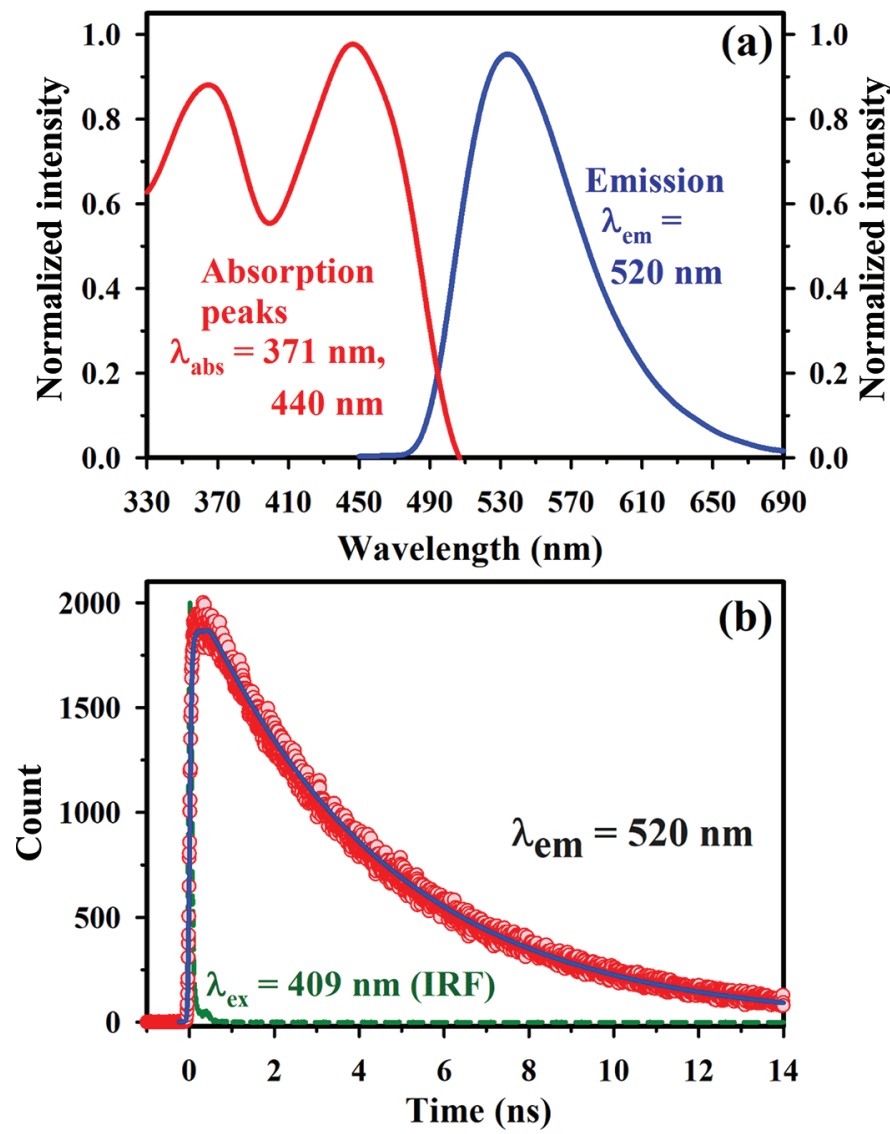

Figure 4. (a) Normalized absorption and emission spectra of riboflavin (RF). (b) Picosecond time-resolved decays of RF. Count $=$ emission count; $\lambda_{\text {ex }}=$ excitation wavelength; $\lambda_{\text {abs }}=$ absorption wavelength $\lambda_{\mathrm{em}}=$ emission wavelength; IRF $=$ instrument response function. to excite the samples. However, earlier reports showed that RF has 2 prominent absorption peaks at 370 and $440 \mathrm{~nm}$ and the emission spectra do not differ much depending on the excitation wavelength (Zhang et al., 2012). We used a 365-nm UV LED for our instrument, which does not contradict our time-resolved results. On the other hand, the faster time component of a few
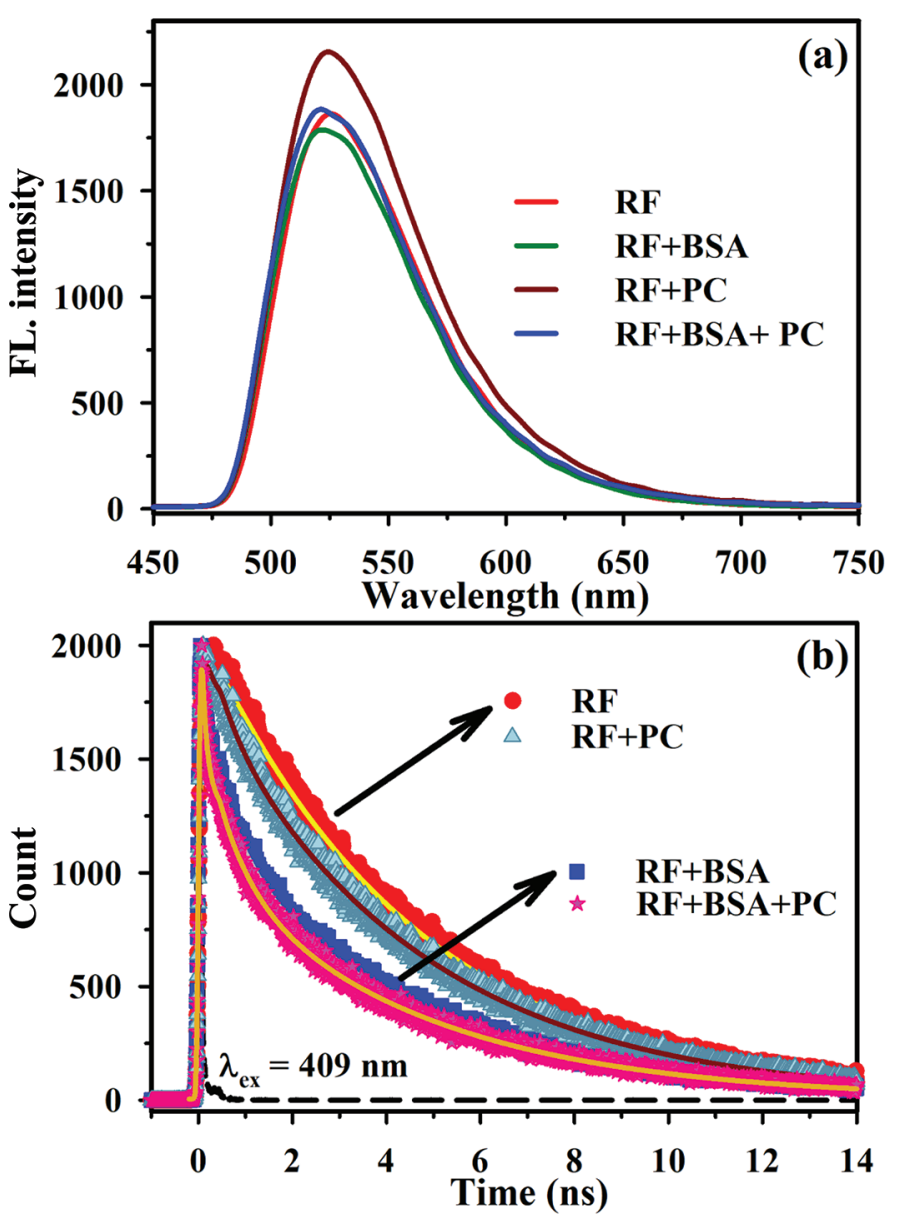

Figure 5. Characteristic changes of riboflavin $(\mathrm{RF})$ in different environments (BSA and $\mathrm{L}-\alpha$-phosphatidylcholine, $\mathrm{PC}$ ) and their mixture examined using (a) emission and (b) picosecond time-resolved decays of RF in those environments. FL. = fluorescence; Count $=$ emission count; $\lambda_{\text {ex }}=$ excitation wavelength. 
hundredths of a picosecond for both cow milk and plant milk does not match to that of pure RF decay time scales. We propose that the interaction of RF molecules with proteins present in the milk samples results in faster decay time (Goswami et al., 2010). From the excited-state lifetime plot of animal and soy milks, it is evident that, compared with animal milk, the protein$\mathrm{RF}$ interaction is higher in soy milk. It is likely that the quantity of the particular protein or the variety of protein present in different milk samples causes quenching of the lifetime of RF molecules. Thus, it is evident from the emission, excitation, and time-resolved fluorescence studies that RF is a potential biomarker to detect milk origin.

To get further evidence of the presence of RF in milk samples of different animal and plant origins, in vitro optical characterization of aqueous RF was carried out. Absorption and fluorescence emission spectra of RF are shown in Figure 4a. Picosecond time-resolved decay is
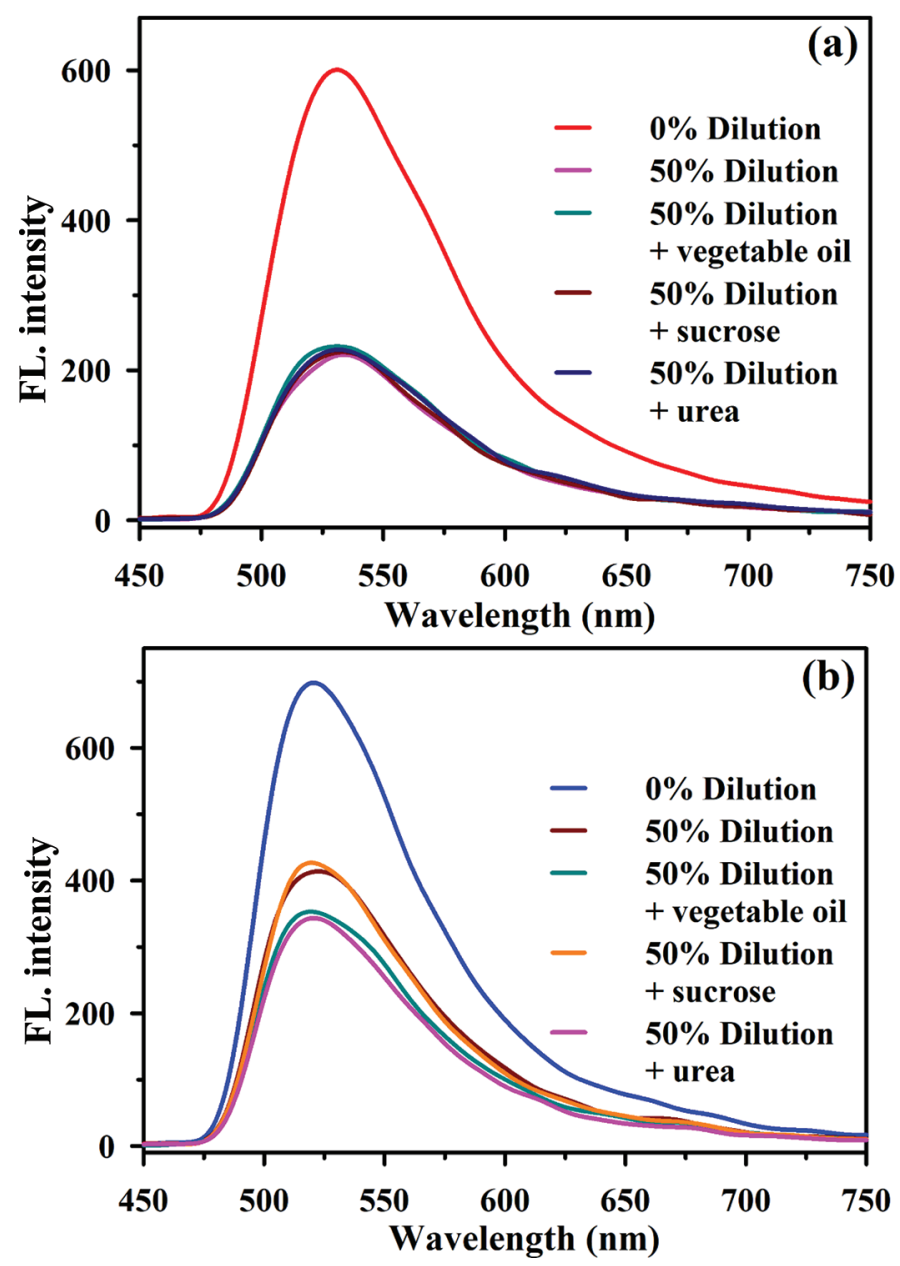

Figure 6. Emission spectra of (a) cow and (b) soy milk in different dilutions and adulteration. FL. = fluorescence. shown in Figure $4 \mathrm{~b}$, and Table 1 provides information about the lifetime of RF molecules. Comparing the optical characteristics of RF shown in Figure 4 with those of the milk samples shown in Figure 3, it can be concluded that RF is the molecule responsible for the optical signature of different milk samples. The longer decay time scales for milk samples and for the RF molecule are observed to be the same.

To rationalize the faster component in the fluorescence decay of milk samples, we examined the behavioral changes of RF in different environments. We chose a protein (BSA) and a lipid (PC) for this purpose. We investigated the changes in the emission of the RF molecule upon addition of BSA and PC to get an idea of the interactions occurring in milk samples; the results are shown in Figure 5a. The interaction of the RF molecule with protein and lipid molecules studied in terms of fluorescence decay time is also shown in Figure 5b. Quenching in excited-state lifetime was observed for
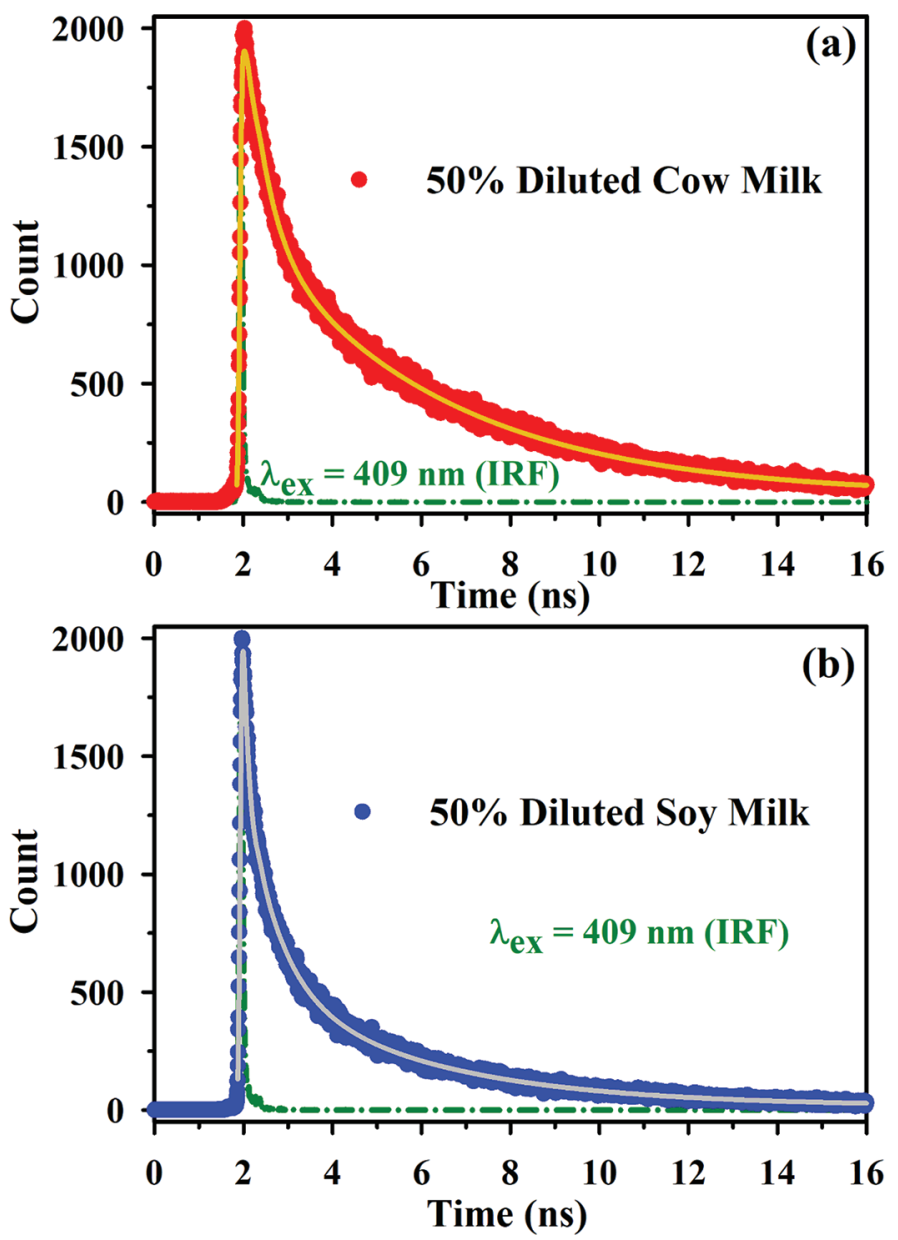

Figure 7. Picosecond time-resolved decays of (a) cow and (b) soy milks in $50 \%$ dilution. Count $=$ emission count; IRF $=$ instrument response function; $\lambda_{\mathrm{ex}}=$ excitation wavelength. 

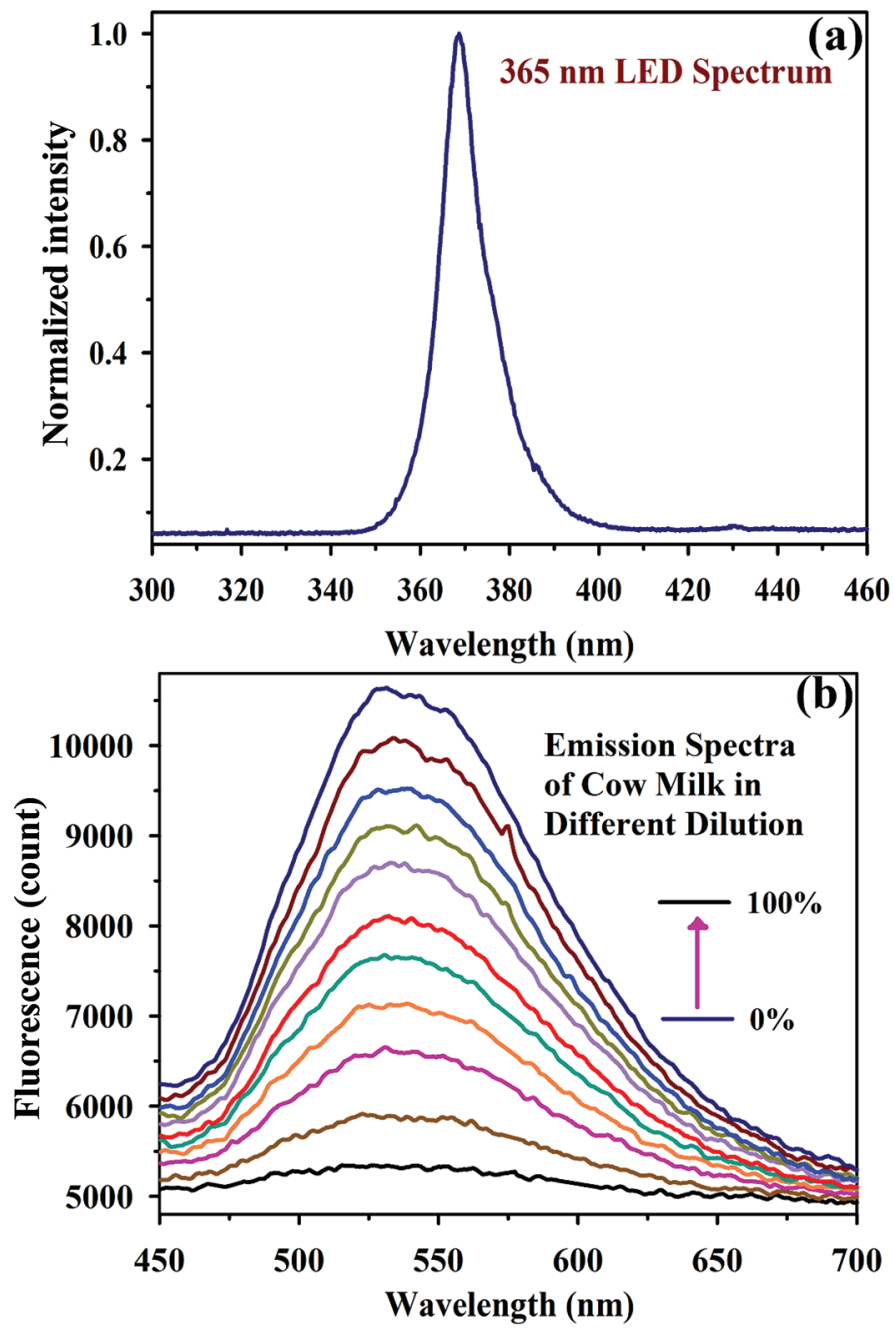

Figure 8. (a) The 365-nm light-emitting diode (LED) spectrum and (b) emission spectra of cow milk in different dilutions in the MilQ-Way instrument. the RF sample upon mixing with BSA. The observation is consistent with the fact that RF undergoes a photo-induced charge transfer reaction in the protein environment (Zhong et al., 2001; Rakshit et al., 2012). On the contrary, no significant quenching occurs for $\mathrm{RF}$ molecules upon interaction with the lipid PC. The probable electron transfer from RF molecules to the AA of the protein BSA could be responsible for the faster time component in the fluorescence decay, whereas no such interaction is feasible in case of RF and the lipid molecule. This observation proves that the faster excited-state time scales observed in cow milk and soy milk, represented in Figure 3c, are due to the interaction of the biomarker RF and the different proteins present in milk samples. The steady-state emission and timeresolved fluorescence of the adulterated milk from animal and plant origins were investigated to find whether the adulterated milk had the same characteristics as the pure milk. The corresponding results are shown in Figures 6 and 7. The decay time scales are summarized in Table 1. Upon dilution, the steady-state emission intensity of both cow milk and soy milk was decreased due to the decreased concentration of the RF molecules present in the milk sample. However, at the same time, the external adulterates has no effect on the emission intensity, which indicates that $\mathrm{RF}$ does not interact with adulterates such as urea, sucrose, or vegetable oil (Figure 6a and b). The excited-state lifetime of the diluted cow milk and soy milk represented in Figure 7a and $\mathrm{b}$ shows that the lifetime of RF molecules present in milk samples was not affected upon dilution. This observation leads to the conclusion that the quenching in the steady-state emission of milk samples is due to dilution of RF concentration and not for any specific interaction, in which case the lifetime would also have
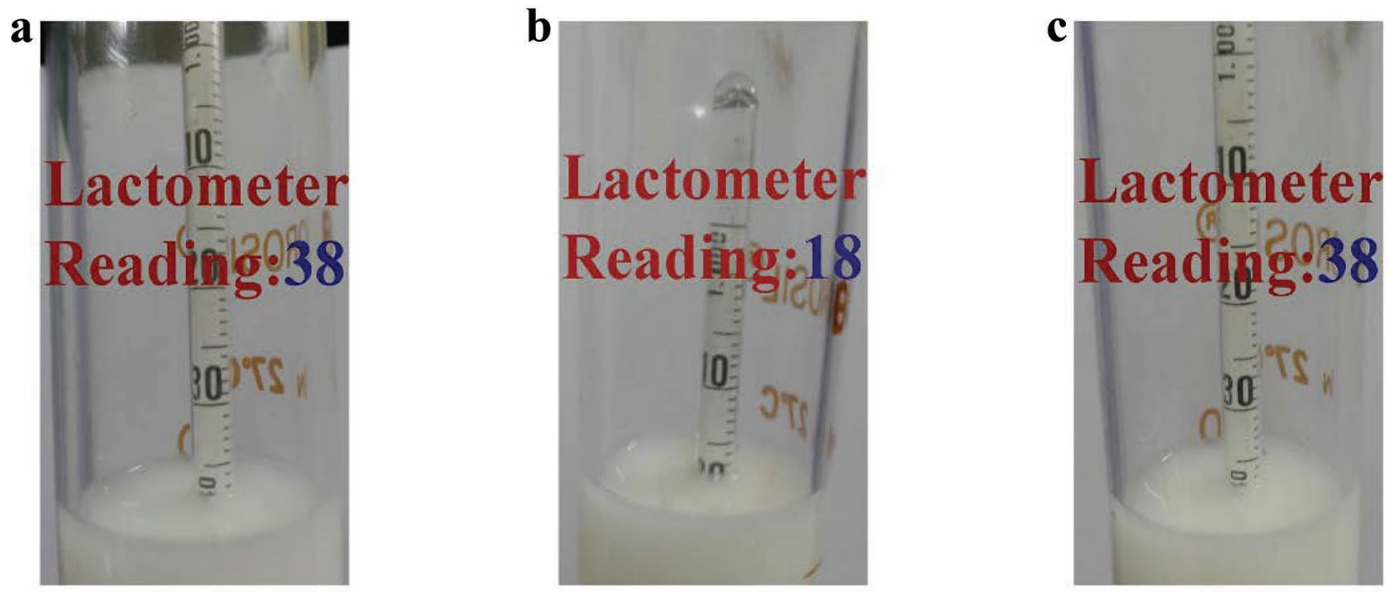

Figure 9. Lactometer readings of local milk (a) before and (b) after dilution with water. Panel c shows the local milk after adulteration with sugar. 


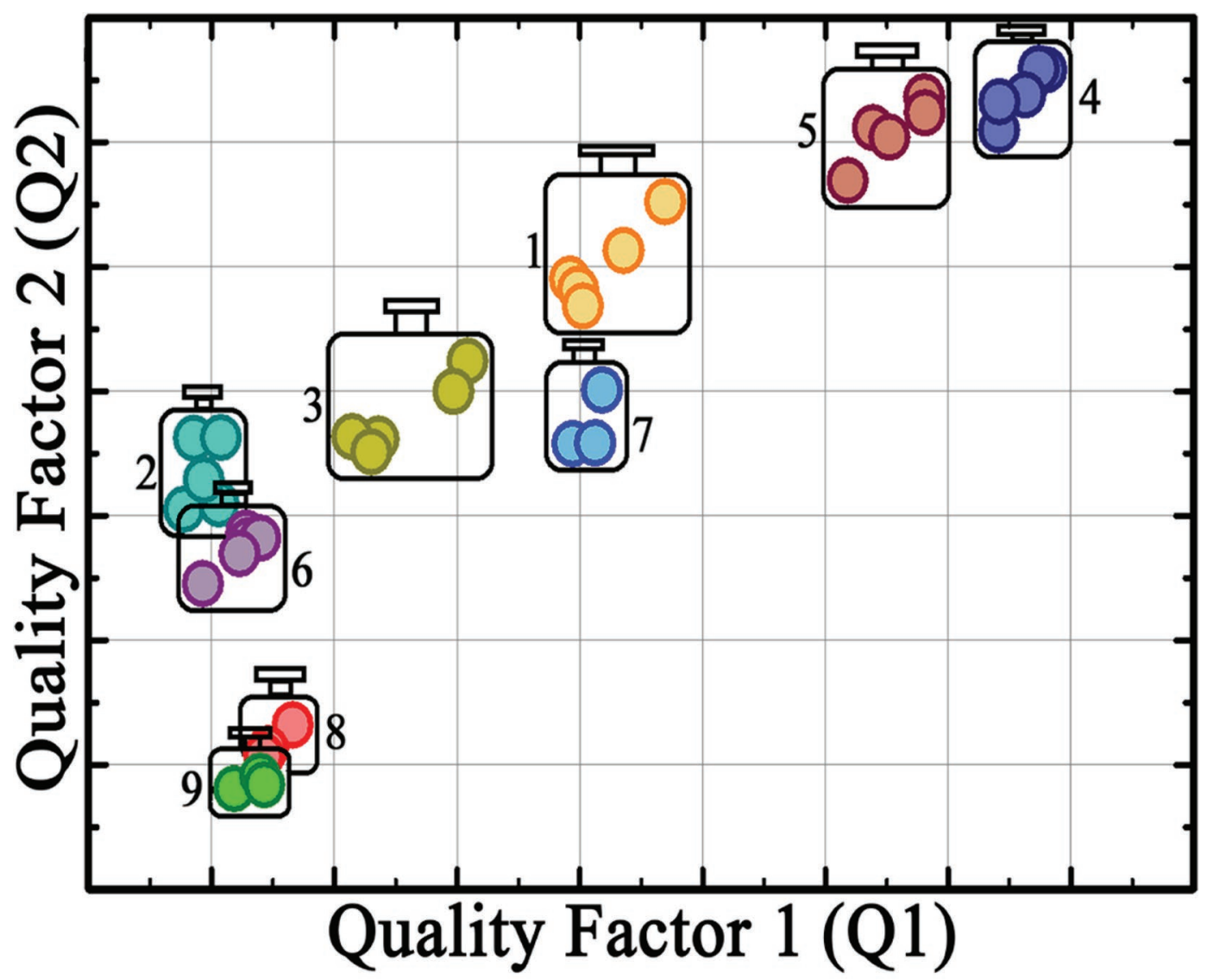

10 Go Daily Milk

20 Nestle a+

$3 \bigcirc$ Soy Milk

$4 \bigcirc$ Amul Standardized Milk
$5 \bigcirc$ Amul Toned Milk

60 Express Dairy

70 Local Milk

$8050 \%$ Diluted Local Milk

\section{0\% Diluted Local Milk with Adulteration}

Figure 10. Mil-Q-Way plot showing 2 quality factors for milks of different commercial origin. Go Daily Milk (Parag Milk Foods Ltd., Andhra Pradesh, India); Nestle a+ (Nestlé India Ltd., Maharashtra, India); Soy Milk (Hershey India Privet Ltd., MP, India); Amul Standardized Milk (Amul, Gujrat, India); Amul Toned Milk (Amul); Express Dairy Milk (Express Dairy Company Ltd., Kolkata, India). Here, the x-axis shows optical density at $365 \mathrm{~nm}$ represents the colloidal density of milk, which is quality factor 1 (Q1), whereas the y-axis shows quality factor 2 (Q2), which is the fluorescence intensity of milk at $520 \mathrm{~nm}$ (excitation at $365 \mathrm{~nm}$ ). Note the coordinate of the local milk (blue) before and after the dilution, which remains unaltered after adulteration.

been affected. Thus, at $50 \%$ dilution, even after giving different types of adulterants, characteristics of pure $(100 \%)$ milk were not changed (data not shown). The first and main step of adulteration is mixing of water. The densitometer is able to detect the dilution of milk. However, if adulterants are added to maintain the concentration, the densitometer fails to detect that. A unique characteristic of our instrument, the Mil-QWay, is that it can distinguish adulteration even after making the SNF of the adulterated milk the same as that in the pure milk. Figure 8a shows the spectra of 365-nm LED, which was used as an excitation source in our instrument. The RF steady-state emission change was monitored in our instrument at different dilutions of a particular type of animal milk, and the results are shown in Figure 8b. For decreased concentration of RF by the addition of water, fluorescence was found to be decreased. Figure 9 shows the lactometer readings of milk from a local vendor. Figure 10 shows the milk library, named Mil-Q-Way Plot. On the basis of the 
emission property of the biomarker RF molecule, which is stable and reliable in different adulterated environments, the library of different types of milks from animal and plant origins from various companies was made using the Mil-Q-Way instrument. It is a 2-parameter plot that includes different milks of various origins and from commercially available sources. Optical density was plotted on the x-axis as Q1, whereas the fluorescence of the sample coming from the RF molecules was plotted in the y-axis as Q2. By comparing the data of a milk sample (no brand) from a local vendor with the data already loaded in the library, the quality of the particular milk can easily be identified using our instrument. The origin of the local milk sample can also be identified using the similar method. We found that the quality of the local milk is close to that of the Go Daily Milk brand. We half-diluted the local milk with water, and the lactometer reading was 38 to 18 (Figure 9a and b). The Q1 and Q2 values are reduced to 0.303 from 0.8163 and to 433,814.64 from 944,557.31 (Figure 10). We added SNF (sucrose) to make the density similar to the original value of 38 (Figure 9c). The prepared sample was considered to be a model of adulterated milk as shown in Figure 10 (sky blue dot). Hence, the location of the sample in the Mil-Q-Way library does not change appreciably, which indicates the signified shift from the original quality of the local milk variety. For evaluation of the model sensitivity and specificity, Bland-Altman analysis (Altman and Bland, 1994) was used. This method gives $\sim 90 \%$ sensitivity and $\sim 100 \%$ specificity in identifying the milk type. Thus, we believe that our instrument provides a much easier solution for detecting milk adulteration than the available high-cost and complicated technologies.

\section{CONCLUSIONS}

In this work, we present a unique spectroscopic strategy for distinguishing different types of milk of different commercial origins even if the SNF of the adulterated milk is manipulated to be the same as that of pure milk. We made a soft library on the basis of the colloidal particles present in the milk and from the fluorescence intensity of the biomarker RF molecules. The positive side of this technology is that the library can be modified per the requirements of the user. Using this strategy, milk quality on a daily basis or in different lactation periods could be analyzed. Also, the packing date of the packed milk could be cross-checked using this technology. Here, different types of milk from different companies have been used and adopted as the standard milks. The origin of milks could be further examined and investigated with different types of milks from different breeds and cattle as well. The technology and instrumentation could be further modified to reduce the manufacturing cost of the instrument.

\section{ACKNOWLEDGMENTS}

We are grateful for financial grants EMR/2016/004698 from the Department of Science and Technology, Science and Engineering Research Board (New Delhi, India) and BT/PR11534/NNT/28/766/2014 from the Department of Biotechnology (New Delhi, India).

\section{REFERENCES}

Altman, D. G., and J. M. Bland. 1994. Diagnostic tests. 1: Sensitivity and specificity. BMJ 308:1552. https://doi.org/10.1136/bmj.308 .6943.1552.

Asensio, L., I. González, T. García, and R. Martín. 2008. Determination of food authenticity by enzyme-linked immunosorbent assay (ELISA). Food Control 19:1-8. https://doi.org/10.1016/j.foodcont 2007.02.010.

Barham, G. S., M. Khaskheli, A. H. Soomro, and Z. A. Nizamani. 2014. Detection and extent of extraneous water and adulteration in milk consumed at Hyderabad, Pakistan. J. Food Nutr. Sci. 2:4752. https://doi.org/10.11648/j.jfns.20140202.15.

Bittante, G., M. Penasa, and A. Cecchinato. 2012. Invited review: Genetics and modeling of milk coagulation properties. J. Dairy Sci. 95:6843-6870. https://doi.org/10.3168/jds.2012-5507.

Borecki, M., M. Szmidt, M. K. Pawłowski, M. Bebłowska, T. Niemiec, and P. Wrzosek. 2009. A method of testing the quality of milk using optical capillaries. Photonics Lett. Pol. 1:37-39.

Chaudhuri, S., S. Sardar, D. Bagchi, S. S. Singha, P. Lemmens, and S. K. Pal. 2015. Sensitization of an endogenous photosensitizer: Electronic spectroscopy of riboflavin in the proximity of semiconductor, insulator, and metal nanoparticles. J. Phys. Chem. A 119:4162-4169. https://doi.org/10.1021/acs.jpca.5b03021.

Chen, R. K., L. W. Chang, Y. Y. Chung, M. H. Lee, and Y. C. Ling. 2004. Quantification of cow milk adulteration in goat milk using high-performance liquid chromatography with electrospray ionization mass spectrometry. Rapid Commun. Mass Spectrom. 18:1167-1171. https://doi.org/10.1002/rcm.1460.

Cheung, P. C. K., and B. M. Mehta. 2015. Handbook of Food Chemistry. Springer-Verlag Berlin Heidelberg, Berlin, Germany.

Cozzolino, R., S. Passalacqua, S. Salemi, P. Malvagna, E. Spina, and D. Garozzo. 2001. Identification of adulteration in milk by matrixassisted laser desorption/ionization time-of-flight mass spectrometry. J. Mass Spectrom. 36:1031-1037. https://doi.org/10.1002/ jms.206.

De Marchi, M., C. C. Fagan, C. O'donnell, A. Cecchinato, R. Dal Zotto, M. Cassandro, M. Penasa, and G. Bittante. 2009. Prediction of coagulation properties, titratable acidity, and $\mathrm{pH}$ of bovine milk using mid-infrared spectroscopy. J. Dairy Sci. 92:423-432. https://doi.org/10.3168/jds.2008-1163.

Goswami, N., A. Makhal, and S. K. Pal. 2010. Toward an alternative intrinsic probe for spectroscopic characterization of a protein. J. Phys. Chem. B 114:15236-15243. https://doi.org/10.1021/ jp105943d.

Handford, C. E., K. Campbell, and C. T. Elliott. 2016. Impacts of milk fraud on food safety and nutrition with special emphasis on developing countries. Compr. Rev. Food Sci. Food Saf. 15:130-142. https://doi.org/10.1111/1541-4337.12181.

Huang, H., H. Yu, H. Xu, and Y. Ying. 2008. Near infrared spectroscopy for on/in-line monitoring of quality in foods and beverages: A review. J. Food Eng. 87:303-313. https://doi.org/10.1016/ j.jfoodeng.2007.12.022. 
Hurley, I. P., R. C. Coleman, H. E. Ireland, and J. H. Williams. 2006. Use of sandwich IgG ELISA for the detection and quantification of adulteration of milk and soft cheese. Int. Dairy J. 16:805-812. https://doi.org/10.1016/j.idairyj.2005.07.009.

Hurley, I. P., H. Elyse Ireland, R. C. Coleman, and J. H. Williams. 2004. Application of immunological methods for the detection of species adulteration in dairy products. Int. J. Food Sci. Technol. 39:873-878. https://doi.org/10.1111/j.1365-2621.2004.00861.x.

Jawaid, S., F. N. Talpur, S. Sherazi, S. M. Nizamani, and A. A. Khaskheli. 2013. Rapid detection of melamine adulteration in dairy milk by SB-ATR-Fourier transform infrared spectroscopy. Food Chem. 141:3066-3071. https://doi.org/10.1016/j.foodchem 2013.05.106.

Lakowicz, J. R. 2013. Principles of Fluorescence Spectroscopy. Springer, New York, NY.

Lipp, M. 1995. Review of methods for the analysis of triglycerides in milk fat: Application for studies of milk quality and adulteration. Food Chem. 54:213-221. https://doi.org/10.1016/0308 -8146(95)00611-L.

Mabrook, M., and M. Petty. 2003. A novel technique for the detection of added water to full fat milk using single frequency admittance measurements. Sens. Actuators B Chem. 96:215-218. https://doi .org/10.1016/S0925-4005(03)00527-6.

Miquel Becker, E., J. Christensen, C. Frederiksen, and V. Haugaard. 2003. Front-face fluorescence spectroscopy and chemometrics in analysis of yogurt: Rapid analysis of riboflavin. J. Dairy Sci. 86:2508-2515. https://doi.org/10.3168/jds.S0022-0302(03)73845 -4 .

Muehlhoff, E., A. Bennett, and D. McMahon. 2013. Milk and Dairy Products in Human Nutrition. Food and Agriculture Organization of the United Nations, Rome, Italy.

Navale, D., and S. Gupta. 2016. Detection of adulteration present in milk products. Int. J. Latest Technol. Eng. Manage. Appl. Sci. 5:103-105.

Ntakatsane, M. P., X. Liu, and P. Zhou. 2013. Rapid detection of milk fat adulteration with vegetable oil by fluorescence spectroscopy. J Dairy Sci. 96:2130-2136. https://doi.org/10.3168/jds.2012-6417.
Poonia, A., A. Jha, R. Sharma, H. B. Singh, A. K. Rai, and N. Sharma. 2017. Detection of adulteration in milk: A review. Int. J. Dairy Technol. 70:23-42. https://doi.org/10.1111/1471-0307.12274.

Powers, H. J. 2003. Riboflavin (vitamin B-2) and health. Am. J. Clin. Nutr. 77:1352-1360. https://doi.org/10.1093/ajcn/77.6.1352.

Pradeep, S., P. Lakshminarayana, R. Varsha, and S. K. Kota. 2016. Screening of adulterants in milk. Int. J. Cur. Res. Rev. 8:25.

Rakshit, S., R. Saha, P. K. Verma, R. K. Mitra, and S. K. Pal. 2012. Ultrafast electron transfer in riboflavin binding protein in macromolecular crowding of nano-sized micelle. Biochimie 94:2673-2680. https://doi.org/10.1016/j.biochi.2012.08.005.

Reddy, Y., M. Laxminaryana, K. Sarma, M. Ranganadham, and K. Shive. 2000. Preparation of a low fat dairy spread using skim milk chakka and butter. Indian J. Dairy Sci. 53:11-14.

Salih, M. A. M., and S. Yang. 2017. Common milk adulteration in developing countries cases study in China and Sudan: A review. J. Adv. Dairy Res. 5:1000192. https://doi.org/10.4172/2329-888X .1000192 .

Ullah, R., S. Khan, H. Ali, M. Bilal, and M. Saleem. 2017. Identification of cow and buffalo milk based on Beta carotene and vitamin-A concentration using fluorescence spectroscopy. PLoS One 12:e0178055. https://doi.org/10.1371/journal.pone.0178055.

Zhang, Y., P. Sukthankar, J. M. Tomich, and G. W. Conrad. 2012. Effect of the synthetic NC-1059 peptide on diffusion of riboflavin across an intact corneal epithelium. Invest. Ophthalmol. Vis. Sci. 53:2620-2629. https://doi.org/10.1167/iovs.12-9537.

Zhong, D., S. K. Pal, C. Wan, and A. H. Zewail. 2001. Femtosecond dynamics of a drug-protein complex: Daunomycin with Apo riboflavin-binding protein. Proc. Natl. Acad. Sci. USA 98:1187311878. https://doi.org/10.1073/pnas.211440298.

\section{ORCIDS}

Samir Kumar Pal @i https://orcid.org/0000-0001-5675-8636 